\title{
Development of a recombinase polymerase amplification combined with a lateral flow dipstick assay for rapid detection of the Mycoplasma bovis
}

\author{
Guimin Zhao', Peili Hou', Yanjun Huan ${ }^{2}$, Chengqiang He ${ }^{1 *}$, Hongmei Wang ${ }^{1 *}$ and Hongbin He ${ }^{1 *}$ (D)
}

\begin{abstract}
Background: Mycoplasma bovis (M. bovis) is a major etiological agent of bovine mycoplasmosis around the world. Point-of-care testing in the field is lacking owing to the requirement for a simple, robust field applicable test that does not require professional laboratory equipment. The recombinase polymerase amplification (RPA) technique has become a promising isothermal DNA amplify assay for use in rapid and low-resource diagnostics.

Results: Here, a method for specific detection of M. bovis DNA was established, which was RPA combined with lateral flow dipstick (LFD). First, the analytical specificity and sensitivity of the RPA primer and LF-probe sets were evaluated. The assay successfully detected $\mathrm{M}$. bovis DNA in $30 \mathrm{~min}$ at $39^{\circ} \mathrm{C}$, with detection limit of 20 copies per reaction, which it was compared the real-time quantitative PCR ( $(P C R$ ) assay. This method was specific because it did not detect a selection of other bacterial pathogens in cattle. Both QPCR and RPA-LFD assays were used to detect M. bovis 442 field samples from 42 different dairy farms in Shandong Province of China, also the established RPA-LFD assay obtained $99.00 \%$ sensitivity, $95.61 \%$ specificity, and 0.902 kappa coefficient compared with the qPCR.

Conclusions: To the author's knowledge, this is the first report using an RPA-FLD assay to visualise and detect M. bovis. Comparative analysis with GPCR indicates the potential of this assay for rapid diagnosis of bovine mycoplasmosis in resource limited settings.
\end{abstract}

Keywords: Mycoplasma bovis, Lateral flow dipstick, Recombinase polymerase amplification, Isothermal nucleic acid amplification, Rapid and visual detection

\section{Background}

Mycoplasma bovis (M. bovis) is a major etiological agent of bovine mycoplasmosis around the world. M. bovis has not only been confirmed as a major pathogen in bovine respiratory disease (BRD), but it has also causes disease in cattle of all ages, such as arthritis, otitis media, mastitis, and reproductive disorders [1]. Due to lack of effectiveness of treatments for controlling the disease in affected herds and decreasing growth rate of the cattle, it can

\footnotetext{
*Correspondence: hchqiang@sdnu.edu.cn; hongmeiwang@sdnu.edu.cn; hongbinhe@sdnu.edu.cn

'Key Laboratory of Animal Resistant Biology of Shandong, Ruminant Disease Research Center, College of Life Science Shandong Normal University, No.88 Wenhua East Road, Lixia District, Jinan 250014, Shandong Province, China Full list of author information is available at the end of the article
}

result in serious economic losses in both dairy and beef cattle herds [2].

In 2008, a severe cattle respiratory disease was reported in Hubei Province of China, thereafter, it quickly spread to over 11 Chinese provinces. The organisms isolated from calf lungs were identified as $M$. bovis and designated strain Hubei-1. The 16S rRNA demonstrated 99.5\% homology with M. bovis type strain PG45 [3]. Other places in China, such as Ningxia, Xinjiang, Guizhou, Chongqing, and Qingdao, later reported $M$. bovis isolated from dairy cows and beef cattle [4]. The lack of an effective control methods to prevent the rapid spread of M. bovis as well as its stubborn persistence on farms requires rapid and accurate diagnosis when clinical signs first appear [1]. 
The development of simple and quick nucleic acid detection methods could greatly improve diagnostics; however, practical on-site testing is infrequently done due to the lack of availability of robustly tested methods. Detection of $M$. bovis from clinical samples by traditional culture methods is quite time-consuming and is often hampered by bacterial contamination. Although detection technology of molecular biology for nucleic acids, such as polymerase chain reaction (PCR) and real-time quantitative PCR (qPCR) has shown high sensitivity and specificity, these methods need professional diagnostic laboratories and thermal cycling device. Such facilities may be lacking in disease epidemic and poor areas, especially in developing countries, the use of rapid

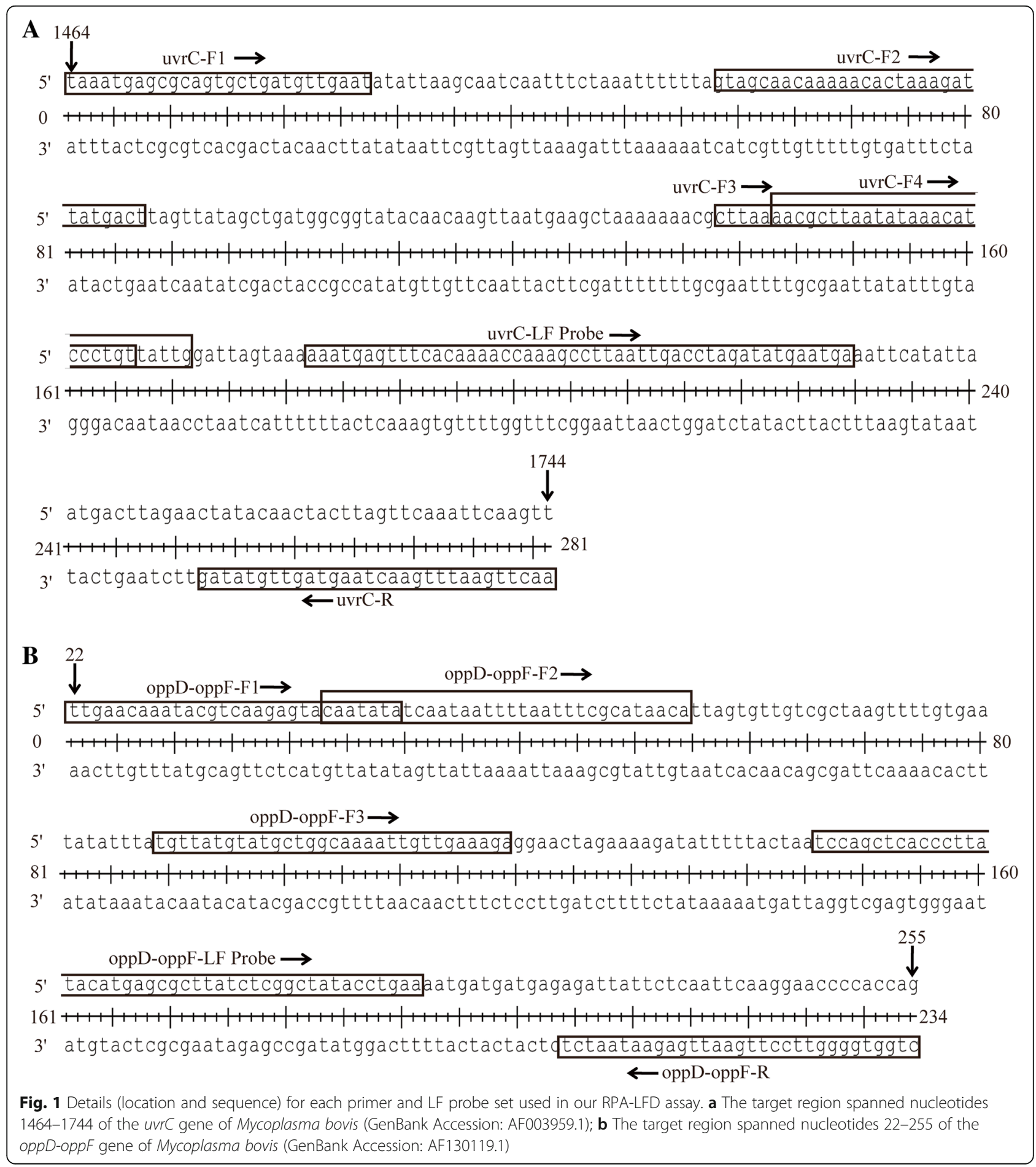


on-site diagnostic methods would be extraordinary helpful in controlling bovine mycoplasmosis.

Recently, for DNA amplification, the recombinase polymerase amplification (RPA) technique has become a promising molecular technology for low-resource, rapid diagnostics [5]. Several types of tests have been described [6], and the RPA combined with lateral flow dipstick (LFD) appears especially suitable for point-of-care diagnosis in clinical specimens. The RPA assay has been reported for the rapid detection of Caprine arthritis-encephalitis virus, Chlamydia trachomatis, Plasmodium falciparum and other pathogens [7-9].

However, the detection of $M$. bovis using RPA-LFD assay has not yet been reported. In this study, we developed a rapid, sensitive, and on-site RPA combined with a LFD assay for the specific detection of M. bovis in the field.

\section{Results}

\section{Evaluation of RPA nfo primer and probe sets}

The analytical specificity of seven primers combinations with LF-probes (Fig. 1 and Table 1) was confirmed using the genomic DNA extracted from $M$. bovis reference type strain PG45. The candidate primers for the RPA-LFD assay was screened with TwistAmp nfo reactions and preliminary analysis was performed on $2 \%$ agarose gel with labeled amplicons. The result showed that primer set uvrC-F1/uvrC-R/uvrC-LF probe yielded specific amplification efficiency for the RPA assay, and produced the expected size of the product of 281 base-pairs (Fig. 2a). The RPA-LFD test line appeared more quickly, within $5 \mathrm{~min}$, and was more distinct than the other sets (Fig. 2b). This set (uvrC-F1/uvrC-R/ uvrC-LF probe) was selected for subsequent evaluation (Fig. 1 and Table 1).

\section{Determination of RPA reaction temperature and time} The results showed that the RPA reaction could be determined at a wide range of temperatures from 30 to $45{ }^{\circ} \mathrm{C}$. In addition, the test band was the brightest between $35^{\circ} \mathrm{C}$ to $42{ }^{\circ} \mathrm{C}$ (Fig. 3a). Therefore, in the later the reaction of RPA-LFD assay temperature was set in $39^{\circ} \mathrm{C}$. Next, the optimum reaction time was estimated between 1 to $35 \mathrm{~min}$. Results indicated that the most distinct band could be seen in the test zone position between 20 to $35 \mathrm{~min}$ with only weak bands seen after $10 \mathrm{~min}$ (Fig. 3b). According to the results, the incubation time was set at $30 \mathrm{~min}$ for the following RPA-LFD testing.

\section{Specificity and sensitivity of the $M$. bovis RPA-LFD assay}

RPA specificity was tested using various Mycoplasma species from cattle and other pathogens that cause respiratory disease and mastitis (Table 2). RPA-LFD revealed high specificity and no cross-reaction was observed against other Mycoplasmas (Mycoplasma agalactiae, Mycoplasma mycoides subsp. mycoides, Mycoplasma bovirhinis, Mycoplasma bovoculi, Mycoplasma bovigenitalium, Mycoplasma dispar, Mycoplasma canadense, Mycoplasma alkalescens, Mycoplasma canis, Mycoplasma arginini), respiratory bacterial pathogens (Pasteurella multocida, Mannheimia haemolytica, Trueperella pyogenes, Histophilus somni, Klebsiella pneumoniae) and mastitis or other pathogens (Staphylococcus aureus, Streptococcus agalactiae, Corynebacterium bovis, Pseudomonas aeruginosa, Proteus mirabilis, Enterobacter aerogenes, Brucella abortus, Escherichia coli) (Additional file 1: Figure S1).

The sensitivity of RPA-LFD and qPCR assay was evaluated, and the repeatability test of limits of detection was showed in Table 3 and Fig. 4. Results from the

Table 1 Primer and probe sequences used for RPA-LFD assay

\begin{tabular}{|c|c|c|c|}
\hline Name & Sequence $\left(5^{\prime}-3^{\prime}\right)$ & Genome location & Amplification size (bp) \\
\hline uvrC-F1 & TAAATGAGCGCAGTGCTGATGTTGAAT & $1464-1490$ & 281 \\
\hline uvrC-F2 & GTAGCAACAAAAACACTAAAGATTATGACT & $1521-1550$ & 224 \\
\hline uvrC-F3 & CTTAAAACGCTTAATATAAACATCCCTGT & $1601-1629$ & 144 \\
\hline uvrC-F4 & AACGCTTAATATAAACATCCCTGTTATTG & $1606-1634$ & 139 \\
\hline uvrC-R & Biotin-AACTTGAATTTGACTAAGTAGTTGTATAG & $1715-1744$ & \\
\hline uvrC-LF Probe & $\begin{array}{l}\text { FAM-AAATGAGTTTCACAAAACCAAAGCCTTAAT } \\
\text { [dSpacer]GACCTAGATATGAATGA-C3 Spacers }\end{array}$ & $1645-1692$ & 100 \\
\hline oppD-oppF-F1 & TTGAACAAATACGTCAAGAGTACAATATA & $22-50$ & 234 \\
\hline oppD-oppF-F2 & CAATATATCAATAATTITAATTTCGCATAACA & $44-75$ & 212 \\
\hline oppD-oppF-F3 & TGTTATGTATGCTGGCAAAATTGTTGAAAGA & $110-140$ & 146 \\
\hline oppD-oppF-R & Biotin-CTGGTGGGGTTCCTTGAATTGAGAATAATCT & $225-255$ & \\
\hline oppD-oppF-LF Probe & $\begin{array}{l}\text { FAM-TCCAGCTCACCCTTATACATGAGCGCTTATC } \\
\text { [dSpacer]CGGCTATACCTGAA-C3 Spacers }\end{array}$ & $167-212$ & 89 \\
\hline
\end{tabular}




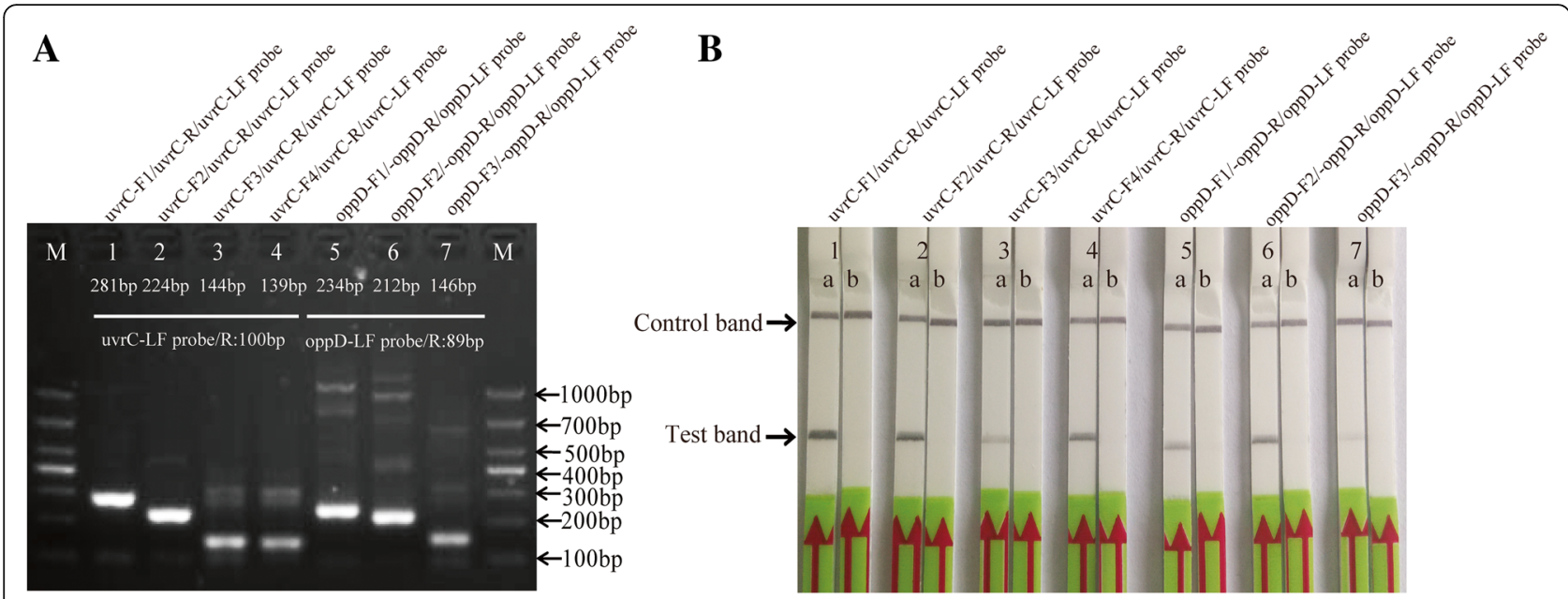

Fig. 2 Screening of Mycoplasma bovis recombinase polymerase amplification combined with a lateral flow dipstick (RPA-LFD) primers and probe. a: The results of RPA-nfo reaction were detected by agarose-gel electrophoresis from seven set of primers and probe combination; b: 'a' showed results of RPA-nfo reaction by LFD test, and DNA template came from extracted Mycoplasma bovis reference type strain PG45 colonies, and 'b' was negative control (DNase-free water) that the corresponding combination of primers and probe. Samples were tested in triplicate with one reaction displayed in figure for each triplicate

testing of serially diluted plasmid DNA showed that RPA-LFD assay was capable of detecting 20 copies/reaction standards DNA, which was 4 times more sensitive than the qPCR assay (Fig. 4).

\section{Performance of RPA-LFD assay on clinical samples}

RPA-LFD performance was analyzed using 442 clinical samples obtained from 42 different dairy farms in Shandong Province, China. The RPA-LFD and qPCR were executed in parallel. The RPA-LFD detected $M$. bovis DNA in 114 of 442 (25.79\%) clinical samples while the qPCR assay found $100(22.62 \%)$ of the same samples positive (Table 4). It indicated both assays were not significantly different using the independent-samples $t$ test $(P>0.05)$. The established RPA-LFD assay yielded 99.00\% sensitivity, 95.61\% specificity, and 0.902 kappa coefficient with the qPCR (Table 5).

\section{Discussion}

Successful surveillance of bovine mycoplasmosis needs a rapid, specific and sensitive diagnostic method. The laboratory-based diagnosis techniques such as qPCR and

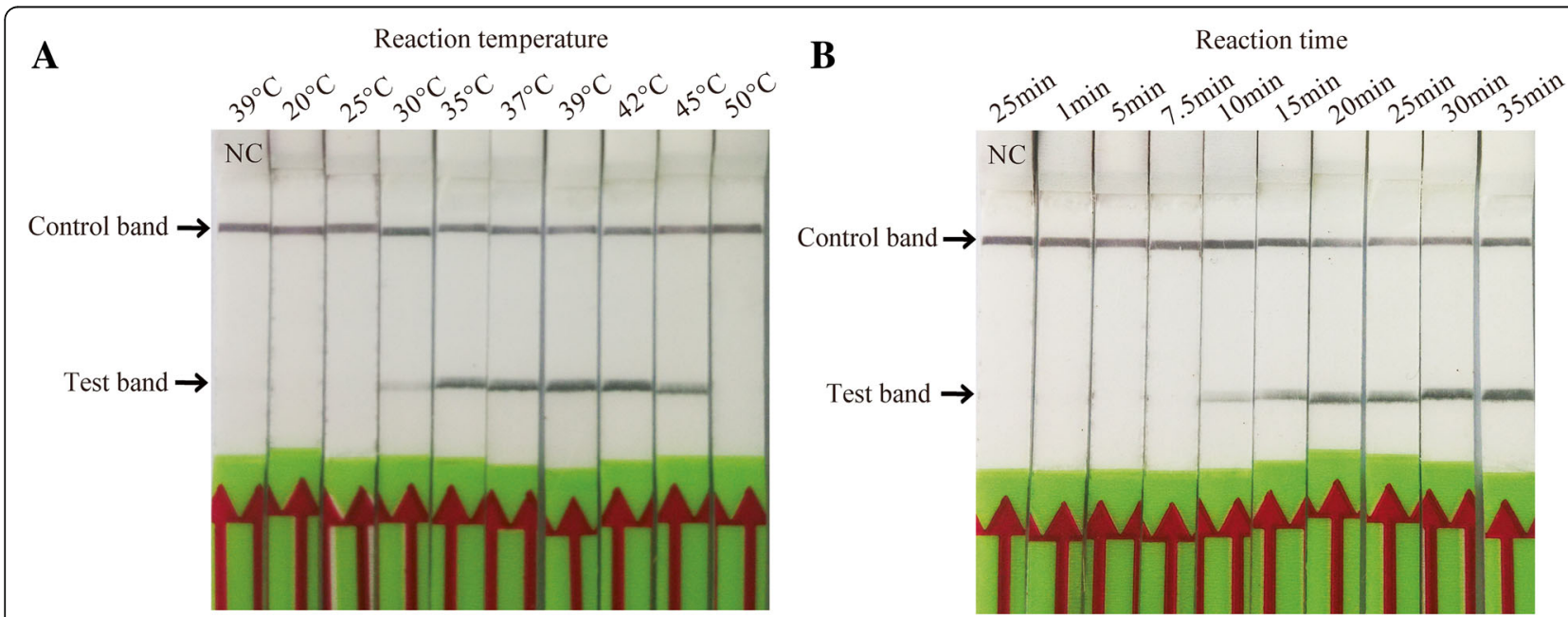

Fig. 3 Optimization of incubation temperature and reaction time for Mycoplasma bovis recombinase polymerase amplification combined with a lateral flow dipstick (RPA-LFD) assay. a The amplification performance of RPA-LFD was effectively within the range of $35^{\circ} \mathrm{C}$ to $45^{\circ} \mathrm{C}$. $\mathbf{b}$ Determination of amplification time. After $10 \mathrm{~min}$ of amplification reaction, the test line was clearly visible on the strip. Lane NC: negative control (DNase-free water). Samples were tested in triplicate with one reaction displayed in figure for each triplicate 
Table 2 Mycoplasma bovis and other bacterial species tested for specificity of the recombinase polymerase amplification combined with a lateral flow dipstick (RPA-LFD) assay

\begin{tabular}{|c|c|c|c|}
\hline Number & Species & Strains/origin & RPA-LFD \\
\hline 1 & Mycoplasma bovis PG45 & ATCC25523a & Positive \\
\hline 2 & Mycoplasma bovis Madison & ATCC27368 ${ }^{\mathrm{a}}$ & Positive \\
\hline 3 & Mycoplasma bovis & ATCC25025 & Positive \\
\hline 4 & Mycoplasma bovis TJ14 & Clinical separation ${ }^{\mathrm{b}}$ & Positive \\
\hline 5 & Mycoplasma bovis SD16 & Clinical separation ${ }^{\mathrm{b}}$ & Positive \\
\hline 6 & Mycoplasma agalactiae PG2 & BNCC132475 & Negative \\
\hline 7 & Mycoplasma mycoides subsp. mycoides & BNCC126186 ${ }^{\mathrm{a}}$ & Negative \\
\hline 8 & Mycoplasma bovirhinis PG43 & ATCC27748 & Negative \\
\hline 9 & Mycoplasma bovoculi & ATCC29104 & Negative \\
\hline 10 & Mycoplasma bovigenitalium & ATCC14173 ${ }^{b}$ & Negative \\
\hline 11 & Mycoplasma dispar & ATCC27140 & Negative \\
\hline 12 & Mycoplasma canadense & ATCC29418 & Negative \\
\hline 13 & Mycoplasma alkalescens & ATCC29103 & Negative \\
\hline 14 & Mycoplasma canis PG14 & ATCC19525 & Negative \\
\hline 15 & Mycoplasma arginini & ATCC23838 ${ }^{\mathrm{a}}$ & Negative \\
\hline 16 & Pasteurella multocida (A) & $\mathrm{BNCC} 126487^{\mathrm{a}}$ & Negative \\
\hline 17 & Mannheimia haemolytica & BNCC128674 & Negative \\
\hline 18 & Trueperella pyogenes & Clinical separation ${ }^{\mathrm{b}}$ & Negative \\
\hline 19 & Histophilus somni & Clinical separation ${ }^{\mathrm{b}}$ & Negative \\
\hline 20 & Klebsiella pneumoniae & BNCC194477 $7^{\mathrm{b}}$ & Negative \\
\hline 21 & Staphylococcus aureus & Clinical separation ${ }^{b}$ & Negative \\
\hline 22 & Streptococcus agalactiae & BNCC185941 & Negative \\
\hline 23 & Brucella abortus A19 vaccine strain & in our laboratory ${ }^{b}$ & Negative \\
\hline 24 & Corynebacterium bovis & BNCC131589 & Negative \\
\hline 25 & Escherichia coli 0157:H7 & BNCC186579 & Negative \\
\hline 26 & Pseudomonas aeruginosa & Clinical separation ${ }^{\mathrm{b}}$ & Negative \\
\hline 27 & Proteus mirabilis & Clinical separation ${ }^{\mathrm{b}}$ & Negative \\
\hline 28 & Enterobacter aerogenes & BNCC $337113^{\mathrm{a}}$ & Negative \\
\hline
\end{tabular}

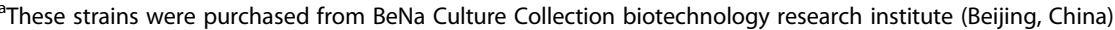

${ }^{\mathrm{b}}$ These strains were preserved in our laboratory

enzyme linked immunosorbent assay (ELISA) are not able to meet the needs of a field test because the equipment required to perform them is not sufficiently portable or robust; furthermore some remote areas lack reliable power. With the development of isothermal nucleic acid amplification method, it is possible to perform on-site diagnostics in resource-limited settings. Here we established a new diagnostic assay based on RPA-LFD and evaluated its applicability to robustly and rapidly identify $M$. bovis, one of the crucial diseases affecting dairy and beef cattle herds in all over the world.

Rapid diagnostic tests for infectious diseases ordinarily use nucleic acid amplification technologies. Molecular tests for $M$. bovis have been developed based on the unique DNA sequences of the $u v r C$ and $o p p D / F$ genes
[10]. Subramaniam et al. (1998) [11] developed a PCR based on the DNA repair $u v r C$ gene, which was shown to clearly differentiate between $M$. bovis and $M$. agalactiae. In this study, the analytical specificity of RPA primers and LF-probes based on $u v r C$ and $o p p D / F$ gene was performed by agarose-gel electrophoresis. At the dSpacer position, when LF-probe is cleaved by nfo nuclease, the probe will be translated into a primer and act as priming for polymerase extension [6]. The one amplified fragment is from the different $\mathrm{F}$ primers and common $\mathrm{R}$ primers, and the other amplified fragment is LF-probe and common $\mathrm{R}$ primers. Therefore, the common $\mathrm{R}$ primers give two bands.

This paper describes a novel RPA-LFD assay based on LF-probe for M. bovis detection. Diagnostic specificity 
Table 3 Results of Mycoplasma bovis plasmid DNA standards detected by real-time qPCR and recombinase polymerase amplification combined with a lateral flow dipstick (RPA-LFD) assay

\begin{tabular}{|c|c|c|c|c|c|c|}
\hline \multirow{2}{*}{$\begin{array}{l}\text { standards DNA } \\
\text { (copies/ul) }\end{array}$} & \multicolumn{3}{|c|}{ Real-time qPCR } & \multicolumn{3}{|c|}{ RPA-LFD } \\
\hline & Ct 1 & Ct 2 & Ct 3 & Test 1 & Test 2 & Test 3 \\
\hline NC & 40.00 & 40.00 & 40.00 & - & - & - \\
\hline $10^{\circ}$ & 40.00 & 40.00 & 40.00 & - & + & - \\
\hline $10^{1}$ & 40.00 & 37.07 & 40.00 & + & + & + \\
\hline $10^{2}$ & 36.76 & 36.79 & 36.41 & + & + & + \\
\hline $10^{3}$ & 33.04 & 32.17 & 32.04 & + & + & + \\
\hline $10^{4}$ & 28.63 & 28.02 & 28.30 & + & + & + \\
\hline $10^{5}$ & 25.24 & 25.12 & 24.94 & + & + & + \\
\hline $10^{6}$ & 20.30 & 20.76 & 20.71 & + & + & + \\
\hline $10^{7}$ & 17.53 & 17.52 & 17.29 & + & + & + \\
\hline
\end{tabular}

NC negative control (DNase-free water)

showed that the assay could detect reference type strain $M$. bovis PG45 and 4 others, but not other pathogens commonly found in cattle. Sensitivity revealed that the RPA-LFD assay was 4 times more sensitive than the qPCR method. To verify the diagnostic suitability of $M$. bovis RPA-LFD assay, the same clinical sample $(n=442)$ set was confirmed by the qPCR assay and yielded 0.902 kappa coefficient with the qPCR.

For diagnostic purposes, several isothermal molecular amplification technologies have been developed in the recent decade [12]. A comparison of 11 isothermal technologies indicates that RPA has some advantages over others. Firstly, RPA reaction is rapid, and nucleic acid amplification can be completed within 10-20 min. Secondly, it operates at lower temperatures $\left(39^{\circ} \mathrm{C}\right)$ than all comparable techniques with the exception of rolling cycle amplification $\left(23{ }^{\circ} \mathrm{C}\right)$ [13]. Several published studies have performed RPA using a simple heat source system. Lillis et al. (2014) demonstrated that incubation of the RPA HIV-1 assay via ambient temperatures or using chemical heaters yields similar results to using electrically powered devices [14]. Moreover, the capacity of RPA to catalyze nucleic acid amplification using only body heat was also demonstrated [15]. A water bath is sufficient to carry out the RPA-LFD testing in the field or in less well-equipped laboratories [16]. Thirdly, commercial availability of freeze-dried reagents makes it easier operation in outside laboratory settings and in remote areas. Moreover, the RPA-LFD provides an easy to read visual signal for clinical point-of-care diagnosis. While PCR needs rapid and accurate temperature control during the amplification cycle, RPA can tolerates temperatures ranging from 35 to $42{ }^{\circ} \mathrm{C}$ without loss of reaction efficiency. This simplifies the instrument and reduces the cost [7]. One disadvantage however is the current price of testing: in China, this is estimated to be $¥ 75 \mathrm{CNY}$ (about 11 USD) for the RPA-LFD and about ¥15 CNY (2 USD) for the qPCR reaction. The RPA-LFD is a new technology and it is believed that the cost will become cheaper as it becomes more widely used.

Further work is clearly necessary for this technology to be taken into the field including using body heat to activate the test and simplified DNA extraction possibly using $\mathrm{NaOH}$ DNA extraction [17].

\section{Conclusions}

An RPA-LFD assay was developed for the rapid detection of $M$. bovis which will be suitable for on-farm testing and in particular in developing countries where sophisticated laboratory equipment is lacking. Its sensitivity and specificity were shown to be comparable to a previously published qPCR.

\section{Methods}

Strains and clinical samples

Reference type strain M. bovis PG45 was cultured as previously described [18], and originally purchased from BeNa culture collection biotechnology research institute (Beijing, China). A total of 442 clinical samples comprising nasal swabs $(n=288)$; fresh lungs $(n=80)$; joint fluids $(n=32)$; and bulk tank milk samples $(n=42)$ were collected between February 2015 and April 2017. Clinical samples from 400 cattle from 42 different dairy farms were examined, and the 42 farms were located in seventeen distinct geographic regions of Shandong province, China [19-21]. Bulk tank milk samples were used to estimate the apparent prevalence of M. bovis infection in 42 different dairy farms. A respiratory disease score was assigned and more than 6 points of cattle have at least two clinical symptoms of respiratory diseases, so they are considered sick [22]. The 288 nasal swab samples taken from all 42 dairy farms in which case suggests after BRD. The 80 fresh lung samples were sampled from postmortem cattle with BRD and the joint fluids 
A

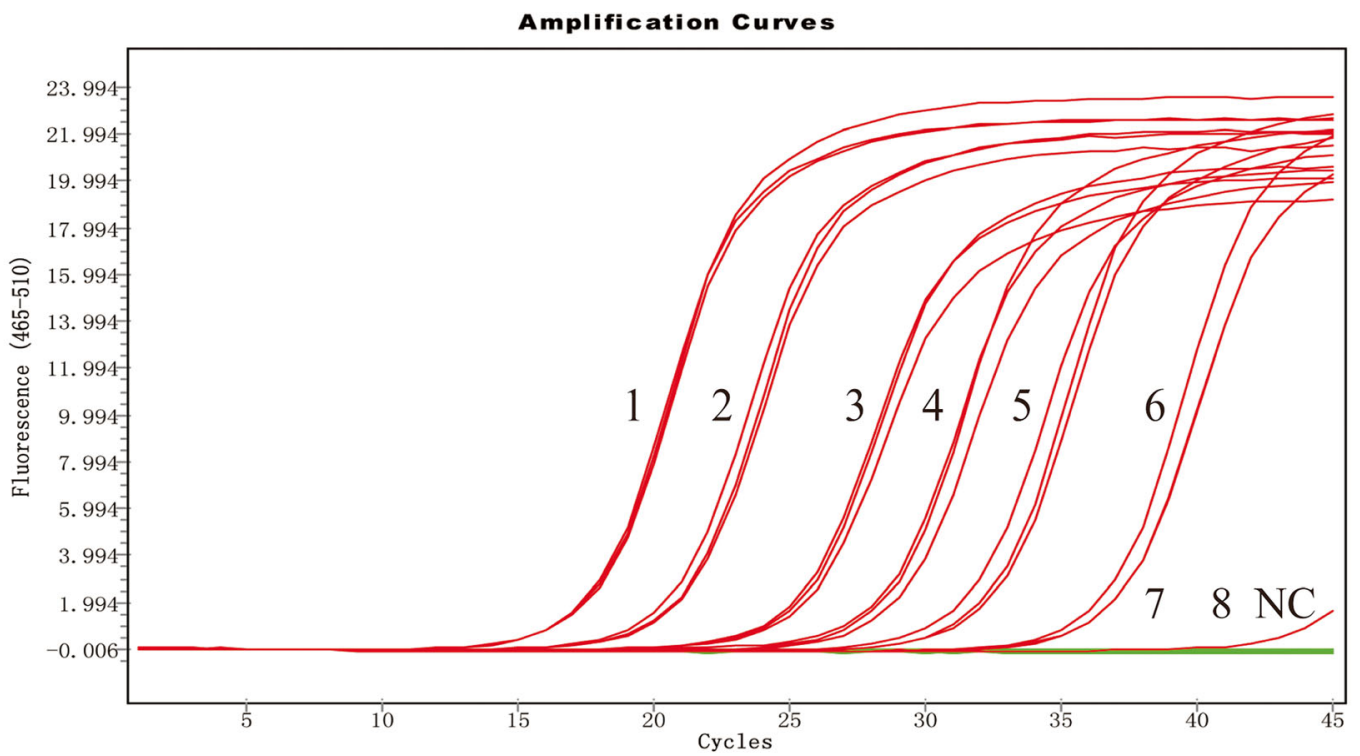

B

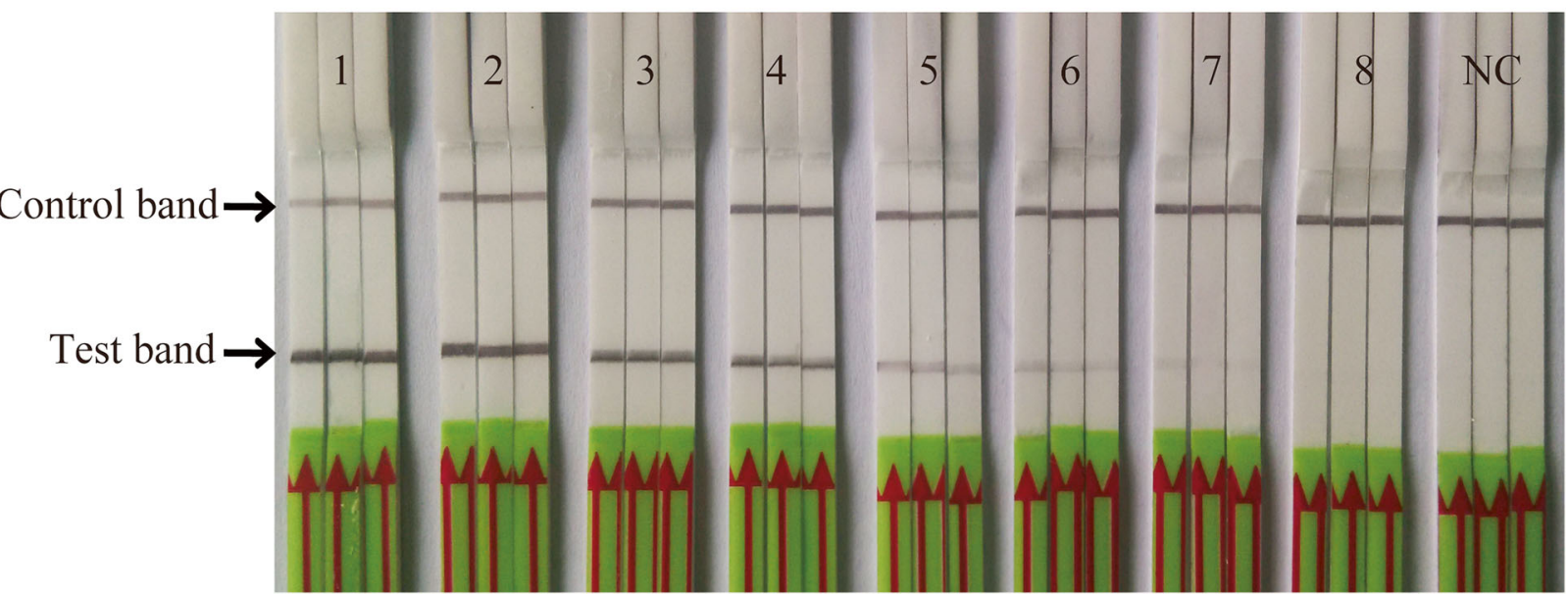

Fig. 4 Comparison of sensitivities of the recombinase polymerase amplification combined with a lateral flow dipstick (RPA-LFD) and Real-time qPCR assays. Molecular sensitivity test results of the two assays were assessed using 10-fold serially diluted DNA as template. a Results by Realtime qPCR (with a detection limit of 83 copies/reaction DNA standards). b Results by RPA-LFD (with a detection limit of 20 copies/reaction DNA standards). Lane 1 to 8: 10-fold serially diluted Mycoplasma bovis plasmid DNA standards from $10^{7}$ to $10^{0}$ copies/uL. Lane NC: negative control (DNase-free water)

Table 4 Camparision of Mycoplasma bovis recombinase polymerase amplification combined with a lateral flow dipstick (RPA-LFD) assay and Real-time qPCR assay on clinical samples

\begin{tabular}{|c|c|c|c|c|c|c|c|}
\hline \multirow[t]{2}{*}{ Samples } & \multirow{2}{*}{$\begin{array}{l}\text { Number } \\
\text { of samples }\end{array}$} & \multicolumn{3}{|l|}{ RPA-LFD } & \multicolumn{3}{|c|}{ Real-time qPCR } \\
\hline & & Positive & Negative & Positive rate (\%) & Positive & Negative & Positive rate (\%) \\
\hline Nasal swabs & 288 & 72 & 216 & 25.00 & 61 & 227 & 21.18 \\
\hline Fresh lungs & 80 & 32 & 48 & 40.00 & 31 & 49 & 38.75 \\
\hline Joint fluids & 32 & 4 & 28 & 12.50 & 4 & 28 & 12.50 \\
\hline Bulk tanks & 42 & 6 & 36 & 14.29 & 4 & 38 & 9.52 \\
\hline Total & 442 & 114 & 328 & 25.79 & 100 & 342 & 22.62 \\
\hline
\end{tabular}


Table 5 Specificity, sensitivity, predictive value and kappa value of recombinase polymerase amplification combined with a lateral flow dipstick (RPA-LFD) and Real-time qPCR assays for diagnosing Mycoplasma bovis infection

\begin{tabular}{|c|c|c|c|c|c|}
\hline & & \multicolumn{2}{|c|}{ Real-time qPCR } & \multirow[b]{2}{*}{ Total } & \\
\hline & & Positive & Negative & & \\
\hline \multirow[t]{5}{*}{ RPA-LFD } & Positive & 99 & 15 & 114 & $92.11 \%(P P V)$ \\
\hline & Negative & 1 & 327 & 328 & $98.78 \%$ (NPV \\
\hline & Total & 100 & 342 & 442 & \\
\hline & & $99.00 \%$ & $95.61 \%$ & 0.902 & \\
\hline & & (Sensitivity) & (Specificity) & (Kappa coefficient) & \\
\hline
\end{tabular}

PPV Positive predictive value, NPV negative predictive value

were collected from 32 calves with arthritis. These clinical samples were tested for $M$. bovis by RPA-LFD and $\mathrm{qPCR}$ assay, in the ruminant disease research center laboratory

\section{DNA extraction}

Approximately 2-4 colonies of the reference type strain M. bovis PG45 were transferred into $200 \mu \mathrm{l}$ of sterile PBS using sterile disposable loop. The genomic DNA was extracted and eluted in $100 \mu \mathrm{l}$ of sterile water by using bacterial genome DNA extraction kit (Tiangen Biotech Co., Ltd., Beijing, China) according to the manufacturer's instructions. Then, DNA was maintained at $20{ }^{\circ} \mathrm{C}$ until screening of the RPA-LFD primer and LF-probe could be performed. The genomic DNA of clinical samples (nasal swabs, fresh lungs and joint fluids) was extracted using TIANamp genomic DNA kit and TIANamp swab DNA kit (Tiangen Biotech Co., Ltd., Beijing, China), specific steps carried out in accordance with the instruction book. The genomic DNA of bulk tank milk samples from the pellet was extracted using TIANamp genomic DNA kit according to reported in literature [23].

\section{RPA primer and LF-probe design}

The primers and LF-probe of the RPA reaction were used to amplify the $u v r C$ gene (nucleotides 1464 to 1744 of the Genbank accession number: AF003959.1) and oppD-oppF gene (nucleotides 22 to 255 of the Genbank accession number: AF130119.1) sequences for $M$. bovis, and seven combinations of candidate primers ( 7 forward and 2 reverse) and two LF-probes were designed according to this two gene sequences. The details were shown in Fig. 1 and Table 1. The analytical specificity was performed using BLAST and no matches with other bacteria.

\section{RPA reaction and lateral flow dipstick (LFD) assay}

Each $50 \mu$ l reaction volume was performed according to manufacturer's instructions (TwistAmp nfo kit), and the volume of primer and LF-probe $(10 \mu \mathrm{M})$ was adjusted accordingly. The following ingredients remained the same: $29.5 \mu \mathrm{l}$ rehydration buffer, $2 \mu \mathrm{l}$ DNA template, and $2.5 \mu \mathrm{l}$ of $280 \mathrm{nM}$ magnesium acetate. The test tubes were incubated at $39{ }^{\circ} \mathrm{C}$ for $25 \mathrm{~min}$ in a thermostatic water tank (Shanghai JingHong laboratory Co., Ltd., China). RPA amplification products were purified and the size of the RPA oligonucleotides was detected by $2 \%$ agarose-gel electrophoresis. Visualization of RPA amplicons was performed using LFD (HybriDetect, Milenia Biotec $\mathrm{GmbH}$, Germany) according to manufacturer's instructions.

\section{RPA conditions and optimization}

To achieve optimal primers and LF-probe, different combinations were analysed for specificity and sensitivity detection. Next, the incubated temperature and time of RPA reaction were assessed according to the reagent instruction (TwistDX); temperature ranges were $20{ }^{\circ} \mathrm{C}$ to $50{ }^{\circ} \mathrm{C}$, and time range was $1 \mathrm{~min}$ to $35 \mathrm{~min}$.

\section{Specificity and sensitivity of the RPA-LFD assay}

The diagnostic specificity of RPA-LFD assay was confirmed with DNA from various reference bacteria strains and strains from clinical cases (Table 2). Most of bacteria species were supplied by BNCC using their recommended media. The forward primer (uvrC-F1): 5'-TAAATGAGCGCAGTGCTGATGTTGAAT-3' and the reverse primer (uvrC-R2): 5'-AACTTGAATTTGAA CTAAGTAGTTGTATAG-3' were used to amplify 281 bp of the $u v r C$ gene of $M$. bovis (1464-1744 of Genbank accession number AF003959.1). The amplified fragment was ligated into plasmid pEASY-T3 cloning vector (Beijing TransGen Biotech Co., Ltd., Beijing, China). The DNA copy number was calculated as described in literature [24]. The analytical sensitivity of the RPA-LFD assay and $\mathrm{qPCR}$ assay was tested on standard plasmid DNA diluted in 10-fold serial steps from $10^{7}$ to 1 copies/ul. For the RPA-LFD assay, in order to evaluate the repeatability limits of detection, the standard plasmid DNA dilutions were tested in duplicates, and this was repeated three times. 


\section{Real-time qPCR assay}

The real-time qPCR assay for $M$. bovis was carried out to amplify a $170 \mathrm{bp}$ sequences between 370 and 538 regions of $u r v C$ gene. The DNA molecular standard was prepared as previously described [5]. The qPCR amplification condition were as previously described $[25,26]$. Briefly, the reaction was prepared as a $20 \mu \mathrm{l}$ reaction volume containing $2 \times$ Probe qPCR Mix, $0.8 \mu$ l of each $25 \mu \mathrm{M}$ Mb-F: 5'-CAAAAGCAAAATGTTAAATTCAG G-3' and Mb-R: 5'-CATATATAAGTGAGACTAACTTA TT-3', $0.8 \mu \mathrm{l}$ of $7.5 \mu \mathrm{M}$ probe: 5'-FAM-CAAAAGCAA AATGTTAAATTCAGG-BHQ2-3' and $2 \mu \mathrm{l}$ of DNA template. The thermal cycling parameters were as previously described $[25,26]$.

\section{Statistical analysis}

Statistical analysis was performed using SPSS 16.0 software (Chicago, IL, USA); also independent-samples $t$ test was used for evaluation of the results. For all analyses, $P<0.05$ was considered significant. The diagnostic performance of the RPA-LFD and qPCR assays was assessed as described previously [27-29]. The kappa coefficient was defined as $(\mathrm{Po}-\mathrm{Pe}) /(1-\mathrm{Pe})[28]$.

\section{Additional file}

Additional files 1: Figure S1. Specificity of the recombinase polymerase amplification combined with a lateral flow dipstick (RPA-LFD) assay. The specificity of the Mycoplasma bovis RPA-LFD assay was assessed for other bacterial pathogens genome cDNA of cattle that present similarly in the clinic. Lanes +: positive control (Mycobacterium bovis PG45), Lane NC: negative control (DNase-free water), Lanes 1: Mycobacterium bovis (Clinical separation), Lanes 2 to 23: Mycoplasma agalactiae, Mycoplasma mycoides subsp. mycoides, Mycoplasma bovirhinis, Mycoplasma bovoculi, Mycoplasma bovigenitalium, Mycoplasma dispar, Mycoplasma canadense, Mycoplasma alkalescens, Mycoplasma canis, Mycoplasma arginini, Pasteurella multocida, Mannheimia haemolytica, Trueperella pyogenes, Histophilus somni, Klebsiella pneumoniae, Staphylococcus aureus, Streptococcus agalactiae, Corynebacterium bovis, Pseudomonas aeruginosa, Proteus mirabilis, Enterobacter aerogenes, Brucella abortus, and Escherichia coli, respectively. Samples were tested in triplicate with one reaction displayed in figure for each triplicate. (DOCX $1581 \mathrm{~kb})$

\section{Abbreviations}

BRD: Bovine respiratory disease; ELISA: Enzyme linked immunosorbent assay; LFD: Lateral flow dipstick; PCR: Polymerase chain reaction; qPCR: Real-time quantitative PCR; RPA: Recombinase polymerase amplification

\section{Acknowledgements}

Not applicable.

\section{Funding}

This work was partially supported by grants from the earmarked fund for the Taishan Scholar and Distinguished Experts (Hongbin He), the National Natural Science Fund of China (31872490, 31672556). Shandong province Key Research \& Development program Fund (2018GNC113011, 2016GNC113006).

\section{Availability of data and materials}

The datasets used and/or analyzed during the current study are available from the corresponding author on reasonable request.

\section{Authors' contributions}

$\mathrm{HBH}$ and GMZ conceived and designed the experiments. GMZ, PLH and YJH performed the experiments. HMW and $\mathrm{CQH}$ analyzed the data. GMZ and $\mathrm{HBH}$ wrote the paper. All the authors read and approved the final manuscript.

\section{Ethics approval and consent to participate}

Experimental protocols for acquiring cattle clinical samples were performed in strict accordance with the Chinese Regulations of Laboratory Animals (Ministry of Science and Technology of People's Republic of China, $20,110,108)$, and the animal study proposal was approved by Shandong Normal University Animal Care and Use Committee (approval No. 20160901).

\section{Consent for publication}

Not applicable.

\section{Competing interests}

The authors declare that they have no competing interests.

\section{Publisher's Note}

Springer Nature remains neutral with regard to jurisdictional claims in published maps and institutional affiliations.

\section{Author details}

'Key Laboratory of Animal Resistant Biology of Shandong, Ruminant Disease Research Center, College of Life Science Shandong Normal University, No.88 Wenhua East Road, Lixia District, Jinan 250014, Shandong Province, China. ${ }^{2}$ College of Animal Science and Technology, Qingdao Agricultural University, No.700 Changcheng Road, Chengyang District, Qingdao 266109, Shandong Province, China.

Received: 8 August 2018 Accepted: 20 November 2018

Published online: 20 December 2018

\section{References}

1. Nicholas RA. Bovine mycoplasmosis: silent and deadly. Vet Rec. 2011;168: 459-62.

2. Nicholas RA, Ayling RD. Mycoplasma bovis: disease, diagnosis, and control. Res Vet Sci. 2003;74:105-12.

3. Li Y, Zheng H, Liu Y, Jiang Y, Xin J, Chen W, et al. The complete genome sequence of mycoplasma bovis strain Hubei-1. PLoS One. 2011;6:e20999.

4. Sun P, Luo H, Zhang X, X J, Guo Y, He S. Whole-Genome Sequence of Mycoplasma bovis Strain Ningxia-1. Genome Announc. 2018;pii: e01367-e01317.

5. Hou P, Wang H, Zhao G, He C, He H. Rapid detection of infectious bovine Rhinotracheitis virus using recombinase polymerase amplification assays. BMC Vet Res. 2017;13:386.

6. Piepenburg O, Williams $\mathrm{CH}$, Stemple DL, Armes NA. DNA detection using recombination proteins. PLoS Biol. 2006;4:e204.

7. Daher RK, Stewart G, Boissinot M, Bergeron MG. Recombinase polymerase amplification for diagnostic applications. Clin Chem. 2016;62:947-58.

8. Kersting S, Rausch V, Bier FF, von Nickisch-Rosenegk M. Rapid detection of plasmodium falciparum with isothermal recombinase polymerase amplification and lateral flow analysis. Malar J. 2014;13:99.

9. Tu PA, Shiu JS, Lee SH, Pang VF, Wang DC, Wang PH. Development of a recombinase polymerase amplification lateral flow dipstick (RPA-LFD) for the field diagnosis of caprine arthritis-encephalitis virus (CAEV) infection. J Virol Methods. 2017;243:98-104.

10. Caswell JL, Bateman KG, Cai HY, Castillo-Alcala F. Mycoplasma bovis in respiratory disease of feedlot cattle. Vet Clin North Am Food Anim Pract. 2010;26:365-79.

11. Subramaniam S, Bergonier D, Poumarat F, Capaul S, Schlatter Y, Nicolet J, et al. Species identification of mycoplasma bovis and mycoplasma agalactiae based on the uvrC genes by PCR. Mol Cell Probes. 1998;12:161-9.

12. James $A$, Macdonald J. Recombinase polymerase amplification. Emergence as a critical molecular technology for rapid, low-resource diagnostics. Expert Rev Mol Diagn. 2015;15:1475-89.

13. Fire $A, X u$ SQ. Rolling replication of short DNA circles. Proc Natl Acad Sci U S A. 1995;92:4641-5.

14. Lillis L, Lehman D, Singhal MC, Cantera J, Singleton J, Labarre P, et al. Noninstrumented incubation of a recombinase polymerase amplification assay for the rapid and sensitive detection of proviral HIV-1 DNA. PLoS One. 2014 9:e108189. 
15. Crannell ZA, Rohrman B, Richards-Kortum R. Equipment-free incubation of recombinase polymerase amplification reactions using body heat. PLoS One. 2014;9:e112146.

16. Yang Y, Qin X, Zhang W, Li Y, Zhang Z. Rapid and specific detection of porcine parvovirus by isothermal recombinase polymerase amplification assays. Mol Cell Probes. 2016;30:300-5.

17. Wang R, Zhang F, Wang L, Qian W, Qian C, Wu J, et al. Instant, visual, and instrument-free method for on-site screening of GTS 40-3-2 soybean based on body-heat triggered recombinase polymerase amplification. Anal Chem. 2017:89:4413-8,

18. Mustafa R, Qi JJ, Ba XL, Chen YY, Hu CM, Liu XL, et al. In vitro quinolones susceptibility analysis of Chinese mycoplasma bovis isolates and their phylogenetic scenarios based upon QRDRs of DNA topoisomerases revealing a unique transition in ParC. Pak Vet J. 2013;33:364-9.

19. Zheng S, Wu X, Zhang L, Xin C, Liu Y, Shi J, et al. The occurrence of porcine circovirus 3 without clinical infection signs in Shandong Province. Transbound Emerg Dis. 2017;64:1337-41.

20. Zheng S, Shi J, Wu X, Peng Z, Xin C, Zhang L, et al. Presence of torque Teno sus virus 1 and 2 in porcine circovirus 3-positive pigs. Transbound Emerg Dis. 2018;65:327-30.

21. He CQ, Liu YX, Wang HM, Hou PL, He HB, Ding NZ. New genetic mechanism, origin and population dynamic of bovine ephemeral fever virus. Vet Microbiol. 2016;182:50-6.

22. Lago A, McGuirk SM, Bennett TB, Cook NB, Nordlund KV. Calf respiratory disease and pen microenvironments in naturally ventilated calf barns in winter. J Dairy Sci. 2006:89:4014-25.

23. McDonald WL, Rawdon TG, Fitzmaurice J, Bolotovski I, Voges H, Humphrey $\mathrm{S}$, et al. Survey of bulk tank milk in New Zealand for mycoplasma bovis, using species-specific nested PCR and culture. N Z Vet J. 2009;57:44-9.

24. Yang Y, Qin X, Wang G, Jin J, Shang Y, Zhang Z. Development of an isothermoal amplification-based assay for rapid visual detection of an Orf virus. Virol J. 2016;13:46.

25. Naikare H, Bruno D, Mahapatra D, Reinisch A, Raleigh R, Sprowls R. Development and evaluation of a novel Taqman real-time PCR assay for rapid detection of mycoplasma bovis: comparison of assay performance with a conventional PCR assay and another Taqman real-time PCR assay. Vet Sci. 2015;2:32-42.

26. Hou P, Zhao G, He C, Wang H, He H. Biopanning of polypeptides binding to bovine ephemeral fever virus G1 protein from phage display peptide library. BMC Vet Res. 2018;14:3.

27. Zhao G, Wang H, Hou P, He C, He H. Rapid visual detection of Mycobacterium avium subsp. paratuberculosis by recombinase polymerase amplification combined with a lateral flow dipstick. J Vet Sci. 2018;19:242-50.

28. Sun $K$, Xing W, Yu X, Fu W, Wang Y, Zou M, et al. Recombinase polymerase amplification combined with a lateral flow dipstick for rapid and visual detection of Schistosoma japonicum. Parasit Vectors. 2016:9:476.

29. Song L, Zhang H, Hou P, Wang H, Zhao G, Xia X, et al. Development and preliminary application of an indirect ELISA to detect infectious bovine Rhinotracheitis virus using recombinant glycoprotein D of IBRV strain SD. Kafkas Univ Vet Fak. 2016:22:503-9.

Ready to submit your research? Choose BMC and benefit from:

- fast, convenient online submission

- thorough peer review by experienced researchers in your field

- rapid publication on acceptance

- support for research data, including large and complex data types

- gold Open Access which fosters wider collaboration and increased citations

- maximum visibility for your research: over $100 \mathrm{M}$ website views per year

At $\mathrm{BMC}$, research is always in progress.

Learn more biomedcentral.com/submissions 